\title{
Analysis of a Single- $\Lambda$ Hypernuclear Event in KEK-PS E373 Nuclear Emulsion Experiment
}

\author{
Myo Thandar Aung ${ }^{1, *}$, Thida Wint ${ }^{1}$, Khin Swe Myint ${ }^{1}$, and Kazuma Nakazawa ${ }^{2}$ \\ ${ }^{1}$ Department of Physics, University of Mandalay, Myanmar \\ ${ }^{2}$ Physics Department, Gifu University, 1-1 Yanagido, Gifu 501-1193, Japan
}

\begin{abstract}
The purpose of this research is to identify a single- $\Lambda$ hypernucleus and its decay products which support to get more information about hyperonnucleon interaction. Before performing the analysis of a single- $\Lambda$ hypernucleus, first, we have deduced the density of emulsion by calibrating the range-energy relation using alpha decay events data from thorium series. It is very important for the mass reconstruction of hypernucleus events in nuclear emulsion. And we have reconstructed a single- $\Lambda$ hypernucleus event by using the kinematical reconstruction for mesonic and non-mesonic decay modes. From the result of our analysis, we can uniquely identify that single- $\Lambda$ hypernucleus is ${ }_{\Lambda}^{9} B$ and decay products are ${ }^{4} \mathrm{He},{ }^{3} \mathrm{He}$, proton and neutron.
\end{abstract}

\section{Introduction}

One of the main goals in hypernuclear physics is to understand the baryon-baryon interaction in a unified model. It is fundamental and important for the study of nuclear physics. To get the complete knowledge of baryon-baryon interaction, it is essential to understand hyperonnucleon (Y-N) and hyperon-hyperon (Y-Y) interactions which can be obtained from single and double hypernuclei respectively. The recent discovery of a neutron star with two solar masses has raised problems of hyperons in the core of neutron stars and Y-N and Y-Y interactions[1].

Nuclear emulsion experiment is one of the suitable methods for the detection of hypernuclear events. Since the measurement using only emulsion in charged particle beam is not effective to search for a very rare reaction, the emulsion counter hybrid experiment, KEK-PS E373, had been developed.Its aim was to study single and double strangeness nuclei via reaction in which $1.66 \mathrm{GeV} / \mathrm{c}$ beam was produced by $12 \mathrm{GeV}$ protons from proton synchrotron on the target made of platinum. The experimental setup is shown in Fig.1.

For E373 experiment, 100 stacks made of $69 l$ emulsion gel were irradiated. Each stack consists of 12 emulsion plates ( 1 thin plate +11 thick plates ) of $24.5 \times 25.0 \mathrm{~cm}^{2}$ area. The thin plate is used as a tracker for the tracking of $\Xi^{-}$particles and the thick plates are used as absorber for the production and analysis of hypernuclear events in emulsion. A stopped $\Xi^{-}$ hyperon is captured by an atom in emulsion. It is firstly captured to an atomic orbit in a highly excited level and transferred into lower orbits emitting Auger electrons and X-rays. Finally, it is completely absorbed by the nucleus and then they form a double hypernucleus, one or two single-hypernuclei, or, if exists, the H-dibaryon. In this experiment, the emulsion analysis

\footnotetext{
*e-mail: myothandaraung.mu@gmail.com
} 
was carried out using a developed automatic scanning with high resolution microscope as shown in Fig. 1.


Figure 1. Right: The setup around the target area of the E373 experiment. Left: Photograph of the microscope system

\section{Analysis}

A photograph and schematic drawing of the event in the upstream plate (\#6) of E373 emulsion stack are shown in Fig. 3. According to the event character, we can conclude that it is single- $\Lambda$ hypernucleus because it has two vertex points. The particle of track \#1, a single hypernucleus, was produced from the beam interaction which a beam particle interacts with one of the nuclei in nuclear emulsion. The particle of track\#1 decayed into three charged particles (track \#2, \#3 and \#4). The measured ranges and angles of these tracks are summarized in table 1.
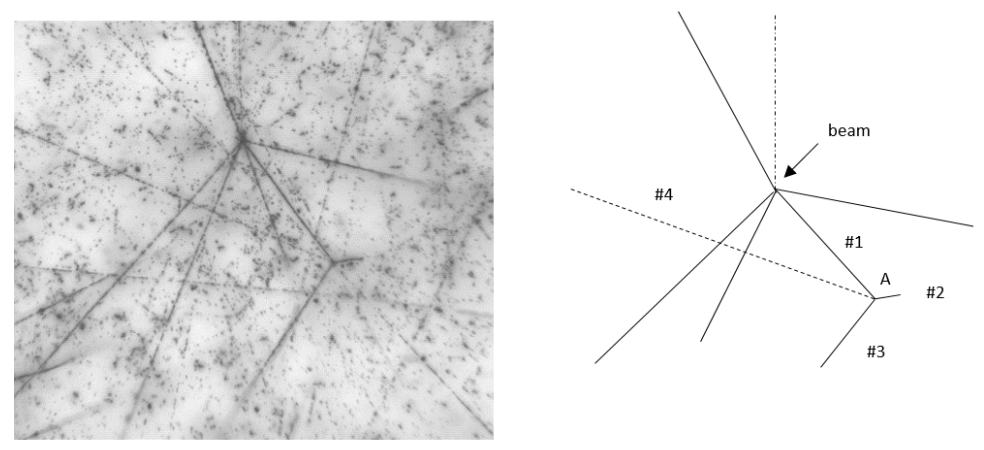

Figure 2. Photograph and schematic drawing of single hypernucleus event

Before performing event analysis, we checked the density of emulsion to calibrate the range-energy relation. It is very important to obtain the density of emulsion in event analysis because it can be changed in different temperature and humidity. In the same emulsion sheet where this event was found, we observed tracks of alpha particles were found to be $23.66 \pm$ $0.57 \mu \mathrm{m}$ for ${ }^{228} \mathrm{Th}$ with known energy of $5.423 \mathrm{MeV}$. Our obtained density value is $3.58 \pm$ $0.50 \mathrm{~g} / \mathrm{cm}^{3}$, which is consistent with $3.66 \pm 0.06 \mathrm{~g} / \mathrm{cm}^{3}$ obtained by a measurement of its size and weight at the time of the beam exposure.

First, in order to analyze the species of single- $\Lambda$ hypernucleus and the decay products, we 
Table 1. The range and angle data of related tracks. Track\#2 and \#3 stopped in the upstram of the emulsion plate and track\#4 ended in the downstream of plate\#6.

\begin{tabular}{llll}
\hline Track\# & Range $(\mu \mathrm{m})$ & theta $(\mathrm{deg})$ & phi $(\mathrm{deg})$ \\
\hline$\# 1$ & $41.55 \pm 0.75$ & $81.70 \pm 0.60$ & $100.38 \pm 0.53$ \\
$\# 2$ & $16.28 \pm 0.25$ & $30.67 \pm 1.14$ & $189.23 \pm 1.73$ \\
$\# 3$ & $544.66 \pm 2.89$ & $115.10 \pm 0.29$ & $54.69 \pm 0.15$ \\
$\# 4$ & $4059.39 \pm 3.66$ & $100.73 \pm 0.02$ & $339.82 \pm 0.12$ \\
\hline
\end{tabular}

Table 2. The comparison of the Q-value and total kinetic energy $\left(E_{\text {total }}\right)$ of non-mesonic decay modes.

\begin{tabular}{llll}
\hline Decay Process & Q-value $(\mathrm{MeV})$ & $E_{\text {total }}(\mathrm{MeV})$ & Remark \\
\hline${ }_{1}^{6} L i \rightarrow p+t+p+n$ & 153.77 & $128.05 \pm 0.44$ & rejected \\
${ }^{9} B \rightarrow{ }^{4} \mathrm{He}+{ }^{3} \mathrm{He}+p+n$ & $\mathbf{1 6 6 . 1 0}$ & $\mathbf{1 6 6 . 8 4} \pm \mathbf{1 . 3 4}$ & acceptable \\
${ }_{\Lambda}^{14} N \rightarrow{ }^{11} B+p+p+n$ & 146.04 & $132.88 \pm 2.54$ & rejected \\
${ }_{\Lambda}^{15} N \rightarrow{ }^{11} B+d+p+n$ & 136.29 & $124.29 \pm 2.42$ & rejected \\
${ }_{\Lambda}^{16} O \rightarrow{ }^{12} \mathrm{C}+d+p+n$ & 146.04 & $158.32 \pm 3.29$ & rejected \\
\hline
\end{tabular}

considered the various possible mesonic decay modes by comparing the sum of the kinetic energies of all emitted charged particles for each decay mode, $E_{v i s}$, with the Q-value which is calculated from the masses of known single hypernuclei [2] together with particles involved in the decay. The kinetic energy of a charged particle was calculated from its measurement ranges by using the range-energy relation.

All mesonic decay modes of single hypernuclei were rejected because the total kinetic energies of emitted charged particles were not comparable with the Q-values. For this event, the coplanarity check is unavailable to know natural particle emission or not because there are three decayed particles from hyperfragment. Therefore, we checked the angle $\alpha$ between the hyperfragment track \#1( $\left.\vec{V}_{H F}\right)$ and the resultant momentum vector $\left(\vec{P}_{\text {total }}\right)$ of three decayed particles by using the formula,

$$
\frac{\vec{V}_{H F} \cdot \vec{P}_{\text {total }}}{\left|\vec{V}_{H F} \| \vec{P}_{\text {total }}\right|}=\cos \alpha
$$

According to the angle check, it is found that the angle $\alpha$ is not equal to zero, i.e. there can be neutron emission. Second, we checked mesonic decay modes with one neutron emission, but all were rejected because Q-values are smaller than the total kinetic energies of all emitted particles including neutron. Third, we considered possible non-mesonic decay modes with one neutron emission by comparing Q-value and total kinetic energy of each decay modes shown in table 2. From this, the most acceptable decay mode is ${ }_{\Lambda}^{9} \mathrm{~B} \rightarrow{ }^{4} \mathrm{He}+{ }^{3} \mathrm{He}+\mathrm{p}+n$ because Q-value is comparable with $E_{\text {total }}$ within error for this decay mode. Our reconstructed mass and binding energy of ${ }_{\Lambda}^{9} B$ are $8580.46 \pm 1.34 \mathrm{MeV} / c^{2}$ and $7.55 \pm 1.34 \mathrm{MeV}$.

\section{Conclusion}

In this research, the result of our analysis is consistent with other previous experimental values $[3,4]$. Therefore, the single- $\Lambda$ hypernucelus, ${ }_{\Lambda}^{9} \mathrm{~B}$, and its decay products, ${ }^{4} \mathrm{He},{ }^{3} \mathrm{He}$, proton and neutron, are uniquely identified. In addition, since one neutron is emitted, the nomination is not easier than the case without neutron emission. 


\section{Acknowledgement}

This research work had been done in collaboration with Prof. Kazuma Nakazawa, spokesperson of E373 experiment, Professor of Physics Department, Gifu University, Japan.

\section{References}

[1] J. S. Bielich, Nucl. Phys. A 804, 309 (2008).

[2] D. H. Davis and J. Pniewiski, Contemp. Phys. 27, 91 (1986).

[3] M. Juric et al., Nucl. Phys. B 52, 1 (1973).

[4] J. Pniewiski et al., Nucl. Phys. A443, 685 (1985). 